\title{
VALORIZAÇÃO DA RESERVA OPERATIVA EM UM AMBIENTE COMPETITIVO PARA GERADORES HIDRELÉTRICOS
}

\author{
Juan Carlos Galvis M* \\ juancgalvisegmail.com \\ Jose Maria Yusta Loyo ${ }^{\dagger}$ \\ jmyusta@unizar.es \\ ${ }^{*}$ Universidade Estadual Paulista \\ LAPSEE-FEIS-UNESP \\ Ilha Solteira, SP, Brasil \\ ${ }^{\dagger}$ Universidade de Zaragoza \\ DEE-CPS-UNIZAR \\ Zaragoza, Espanha
}

Antonio Padilha Feltrin*

padilhaddee.feis.unesp.br

\begin{abstract}
Operating Reserve Pricing of Hydraulic Generators in a Competitive Framework.

A pricing proposal for operating reserve ancillary services in a hydraulic competitive framework is presented. In this framework, generator agents bid for the availability of the service. This proposal aims the efficient reserve pricing based on both, agents competition and system operating conditions. In the first part of this paper, some aspects concerning to operating reserve are described, focusing, particularly, in the availability, use and opportunity costs. In the second part, the proposal is described, showing the market structure, the type of auctions and a joint power and operating reserve dispatch model, that considers the hydraulic and electrical characteristics of the system. These considerations are important in the Brazilian system, where there is a high percentage of hydraulic generation, that needs to be coordinated in a centralized manner, optimizing the operational costs. The problem is modeled with a linear integer mixed formulation, and is solved using a commercial optimization solver. The methodology is applied on a three
\end{abstract}

Artigo submetido em 04/12/2009 (Id.: 01091)

Revisado em 15/02/2010, 15/03/2010

Aceito sob recomendação do Editor Associado Prof. Julio Cesar Stacchini Souza bus, three generator system for illustrative purposes.

KEYWORDS: Ancillary services, Operating reserve, Competitive framework, Availability, Hydraulic generation.

\section{RESUMO}

Este trabalho apresenta uma proposta para a valorização dos serviços ancilares de reserva operativa, sob um esquema competitivo composto por geradores hidrelétricos. Nesse esquema, os geradores ofertam pela disponibilidade do serviço. Esta proposta busca atribuir um valor à reserva de forma eficiente, em função da concorrência entre os agentes e as condições de operação do sistema. Na primeira parte do artigo, são descritos diversos aspectos relacionados com a reserva operativa destacando, particularmente, os componentes de custo de disponibilidade, uso e oportunidade destes serviços. Na segunda parte é apresentada a proposta propriamente dita, indicando a estrutura do mercado, o tipo de ofertas, assim como um modelo de despacho hidrelétrico conjunto de potência e reserva, que considera as restrições hidráulicas e elétricas do sistema. Estas considerações são importantes no caso do Sistema Brasileiro, já que existe uma alta porcentagem de produção hidrelétrica, cuja operação precisa ser coordenada de forma centralizada, visando minimizar os custos operacionais. O problema 
de despacho é modelado através de uma formulação linear inteira mista, e é resolvido usando um solver de otimização comercial. A proposta é aplicada em um sistema de três barras e três geradores com fins ilustrativos.

PALAVRAS-CHAVE: Serviços ancilares, Reserva operativa, Esquema competitivo, Disponibilidade, Produção hidrelétrica.

\section{INTRODUÇÃO}

A desverticalização do modelo econômico trouxe mudanças significativas no modelo tarifário dos sistemas de energia elétrica. Assim, atividades como a geração e a comercialização de energia migraram de um cenário monopólico para um ambiente competitivo. Esse processo de reestruturação foi realizado para aumentar a eficiência no setor e incentivar o investimento na expansão do sistema (Rudnick et al., 2008). Entre os problemas que apareceram na indústria elétrica por causa da desverticalização se podem mencionar: a divisão de responsabilidades, a definição de serviços, a valoração e atribuição de custos; etc. Dentro desta problemática se encontra a valorização de serviços ancilares, os quais são serviços fornecidos por agentes conectados ao sistema, com a finalidade de garantir a transmissão de energia elétrica sob condições de qualidade, confiabilidade e segurança (Hirst and Kirby, 1996).

No mundo, existem diversos serviços classificados como ancilares. No caso do sistema brasileiro, a Agencia Nacional de Energia Elétrica - ANEEL, definiu o seguinte conjunto de serviços (ANNEL, 2003; ANNEL, 2007):

- controle de frequência primário e a reserva associada;

- controle de frequência secundário e a reserva associada;

- reserva de prontidão;

- suporte de reativo;

- auto-restabelecimento;

- sistema especial de proteções.

As reservas associadas ao controle de frequência primário e secundário, e a reserva de prontidão, fazem parte da Reserva de Potência Operativa - RPO, isto é, a reserva que é utilizada na operação em tempo real para garantir a operação segura e confiável do sistema.

Neste trabalho é apresentada uma proposta para a valorização da RPO (especificamente as reservas utilizadas para o controle de frequência), considerando um cenário de concorrência e várias características do sistema brasileiro.
Na primeira parte do documento (seções 2 e 3) são tratados diversos aspectos relacionados com a reserva operativa. $\mathrm{Na}$ seção 2 é apresentada uma revisão bibliográfica referente aos métodos de aquisição e valorização da reserva. Na seção 3 , se definem as reservas para regulação de frequência e os componentes de custos associados. Na segunda parte do trabalho (seções 4 e 5) é descrita a proposta de valorização da reserva operativa. Na seção 4, se descreve o método de valorização, enquanto na seção 5 se mostra a aplicação do método através de um exemplo teórico. Finalmente, na seção 6, são apresentadas as conclusões do trabalho.

\section{REVISÃO BIBLIOGRÁFICA}

A reserva operativa é classificada de diversas formas no mundo, dependendo tanto das características e necessidades de cada sistema, quanto dos critérios de confiabilidade e segurança adotados. Uma descrição da classificação e das características técnicas da reserva em diversos países é apresentada em (Rebours et al., 2007). Além das diferenças técnicas, existem vários mecanismos para a aquisição e valorização da reserva (Rebours et al., 2007a), os quais podem ser diferenciados em função do grau de concorrência (Rebours et al., 2007b).

A valorização do serviço pode ser abordada considerando um cenário de mercado ou de não mercado. Num cenário de mercado ou cenário competitivo, se oferece aos agentes a liberdade de determinar o valor do serviço estabelecendo, sob determinadas regras de concorrência, o preço de aquisição. No cenário de não mercado, o valor do serviço é geralmente determinado pela entidade reguladora, a partir de estudos de planejamento e operação do sistema. Portanto, é usual determinar uma fórmula tarifária e/ou estabelecer um processo de contratação bilateral entre a parte solicitante (Operador Independente do Sistema OIS) e a parte fornecedora do serviço. Alguns países têm implementado esquemas competitivos (mercados de curtíssimo prazo/leilões) para a aquisição destes serviços, enquanto outros tem optado por utilizar um esquema de tarifa regulada ou a implementação de contratos bilaterais. Encontram-se, também, situações nas quais alguns serviços são impostos e não remunerados.

Dentro dos esquemas competitivos e, para o caso da reserva operativa, existem basicamente duas formas de otimização: sequencial e cootimizada (Kirschen and Strbac, 2004). No esquema sequencial, o mercado de energia e o(s) mercado(s) de reserva são resolvidos um após o outro, em uma forma sequencial, enquanto no esquema cootimizado, todos estes mercados são resolvidos simultaneamente. (González et al., 2007) referenciam vários dos trabalhos que utilizam esses esquemas e estabelecem uma classificação em função de quem realiza a otimização (o Operador Independente do 
Sistema - OIS, ou o gerador $\left.{ }^{1}\right)$.

Por outro lado, algumas das pesquisas realizadas no Brasil, referentes à valorização dos serviços de reserva de potência ativa considerando um cenário de não mercado, são: (Sousa et al., 2004), (Ribeiro, 2005) e (Velasco, 2004). (Sousa et al., 2004) abordam a valorização da reserva do ponto de vista da perda de eficiência na geração devido ao fornecimento do serviço, enquanto (Ribeiro, 2005) valoriza o serviço em termos do custo evitado de interrupções do sistema, no caso que o serviço não fosse fornecido. (Velasco, 2004) apresenta uma revisão dos custos associados ao fornecimento de reserva e diferencia a valorização da reserva de potência ativa, utilizada para controle de frequência, da valorização da reserva de planejamento. A primeira é valorizada em termos de índices de eficiência na regulação de frequência, enquanto a segunda é valorada em termos de índices de confiabilidade.

Com relação às pesquisas que consideram um cenário de mercado, alguns trabalhos realizados no Brasil são: (Silva, 2005), (Agurto, 2004), (Costa, 2004) e (Schier, 2007). (Silva, 2005) aborda a valorização do ponto de vista do OIS $^{2}$. A proposta considera as hipóteses clássicas de competição perfeita e está baseada na estrutura do modelo implementado na Califórnia (substituição de serviços). (Agurto, 2004) e (Costa, 2004) apresentam um modelo de otimização conjunta de energia e reserva operativa considerando um esquema de mercado diário. (Schier, 2007) compara diversas métodos de otimização sequencial.

Diferentemente dos trabalhos anteriores, este trabalho propõe uma estrutura de valorização em um cenário competitivo para geradores hidrelétricos, considerando alguns aspectos do sistema brasileiro.

Atualmente, no Brasil, a reserva é adquirida utilizando o mecanismo de contratação bilateral, denominado Contrato de Prestação de Serviços Ancilares - CPSA. Esse contrato é realizado entre o ONS e o gerador, e estabelece os termos e condições gerais para a prestação dos serviços ancilares definidos no sistema brasileiro, indicando quais unidades estão destinadas ao fornecimento desses serviços (ONS, 2003b). O contrato é de longa duração e termina quando acaba o direito de concessão da empresa geradora ou quando uma das partes o requer. No referente aos serviços de controle de frequência, neste contrato unicamente é considerado o controle secundário de frequência, indicando que a prestação do serviço não representa nenhum ônus para o consumidor, e que os custos de implantação, operação e manutenção serão ressarcidos via Encargos de Serviços do

\footnotetext{
${ }^{1} \mathrm{O}$ OIS geralmente busca minimizar o custo de aquisição, enquanto o gerador busca minimizar o risco frente ao mercado ou maximizar o rendimento.

${ }^{2}$ No caso do Brasil, o Operador Nacional do Sistema Elétrico - ONS.
}

Sistema ${ }^{3}$.

Diferentemente do serviço de suporte de reativo, o serviço de controle de frequência não tem definido atualmente uma tarifa de serviços ancilares - TSA.

\section{RESERVAS PARA REgULAÇÃo DE FREQUÊNCIA}

Embora a RPO abranja diversas classes de reserva, neste trabalho serão consideradas apenas as reservas para regulação de frequência. Essas reservas são classificadas de diversas formas, sendo usual diferenciar três tipos, como é descrito a seguir (Rebours et al., 2007):

Reserva para regulação primária: Permite compensar as variações (aumentos ou diminuições) da geração com relação à carga na operação em tempo real. A regulação primária possui um tempo de resposta rápido, da ordem de alguns segundos, e um tempo de uso da ordem de alguns minutos dependendo da duração dessas variações. A ação de regulação primária é realizada através do regulador de velocidade das unidades geradoras.

Reserva para regulação secundária: Esta reserva é usualmente utilizada para o Controle Automático de Geração - CAG (Rebours et al., 2007), o qual é um controle centralizado que corrige os desvios de frequência e os intercâmbios programados entre as áreas do sistema (Jaleeli et al., 1992). O tempo de resposta da regulação secundária é de algumas dezenas de segundos até minutos, dependendo da tecnologia de geração, enquanto o tempo de uso depende da duração do desvio.

Reserva para regulação terciária: Corresponde à reserva utilizada para resguardar ou restituir a reserva para regulação primária e secundária de frequência (Rebours et al., 2007). O tempo de resposta da regulação terciária é da ordem de alguns minutos e maior do que o tempo de resposta da regulação secundária, enquanto o tempo de uso depende da duração das variações de geração em relação à carga. A regulação terciária usualmente é realizada através de modificações manuais no despacho, ou por meio do chaveamento de unidades de reserva. No caso do sistema brasileiro, a alocação da reserva para regulação terciária se realiza, unicamente, quando a demanda de reserva do sistema (calculada probabilisticamente) ultrapassa $5 \%$ da demanda de potência ativa (ONS, 2008b). De acordo com os procedimentos técnicos do operador de rede, essa reserva é distribuída, de preferência, entre todas as unidades conectadas ao CAG.

\footnotetext{
${ }^{3}$ Custos incorridos na manutenção da confi abilidade e estabilidade do sistema e que não estão incluídos no processo de liquidação comercial da energia.
} 
Neste trabalho, são adotadas as mesmas definições do ONS, no referente às reservas de regulação primária, secundária e terciária, e é desconsiderada a reserva de prontidão, a qual é também denominada reserva complementar. Essa reserva está associada às unidades térmicas, as quais estão fora do escopo deste trabalho.

\subsection{Componentes de custo}

Uma classificação dos custos da RPO é realizada na figura 1 .

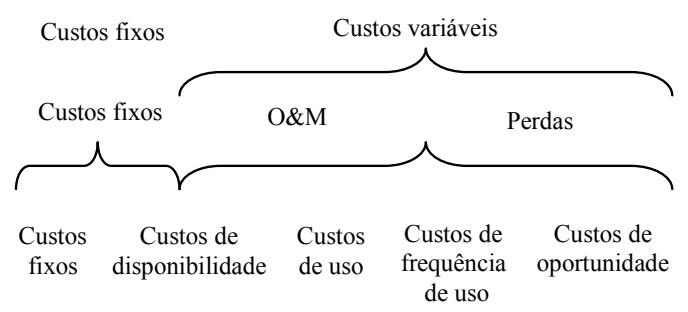

Figura 1: Componentes de custos da RPO.

Na figura 1, os custos fixos representam a parcela que não depende da quantidade fornecida, a diferença dos custos variáveis, que dependem da produção. No sistema brasileiro, para o caso dos serviços ancilares, os custos variáveis são discriminados em custos de operação e manutenção - O\&M, e custos por perdas adicionais (ONS, 2003a). Estes custos podem ser desagregados de uma forma mais específica conforme é ilustrado. Pode-se observar que o componente de custo de disponibilidade possui duas parcelas, uma fixa e outra variável. Neste trabalho, considera-se que a reserva é valorizada por meio dos componentes de custos apresentados a seguir.

\subsubsection{Custo fixo}

Esta parcela representa o investimento no equipamento necessário para o fornecimento do serviço e refere-se a:

- Custos de adequação dos equipamentos para fornecimento de reserva, os quais estão geralmente associados às usinas que possuem equipamentos antigos;

- Custos de investimento no sistema administrativo, de medição, controle, comunicação e processamento de dados;

- Custos de investimento em equipamentos de regulação. Cabe destacar que as usinas antigas já têm incluída esta parcela no custo fixo de geração e, neste sentido, deve ser realizada uma revisão dos custos, para determinar se esse componente continua sendo parte do custo de produção, ou se é conveniente separá-lo e remunerá-lo como um custo fixo por serviços ancilares de reserva, levando em conta que, provavelmente, o investimento já foi recuperado. No caso de usinas novas, esse custo deveria ser diretamente incluído na valorização do serviço ancilar.

Além dos custos anteriores, existe o custo de investimento em capacidade de reserva ou reserva planejada (Velasco, 2004). A reserva planejada é definida como a folga de geração adicional que não é utilizada no curto prazo. O mecanismo de remuneração dessa reserva é denominado pagamento por capacidade, e visa garantir um nível apropriado de investimento em geração elétrica. No sistema brasileiro, o pagamento não existe, por considerar que essa parcela faz parte do custo fixo de geração, o qual, já é recuperado com a venda de energia. Como a reserva considerada neste trabalho corresponde a reserva de potência operativa, este componente de custo é desconsiderado.

\subsubsection{Custos de disponibilidade}

Para todos os tipos de reserva operativa, os custos de disponibilidade, fixos e variáveis, se referem aos custos adicionais em que incorre o agente para manter disponível o serviço. Esses custos são descritos a seguir.

- Custos de manutenção: a medição da parcela de manutenção é complexa, já que as usinas dispõem dos custos de manutenção totais e não dos custos de manutenção individualizados. Se dentro de uma usina, um grupo de unidades fosse destinado unicamente para gerar energia, e outro grupo de máquinas fosse destinado para gerar energia e fornecer reserva, a diferença dos custos de manutenção dos dois grupos, indicaria os sobrecustos nos quais o agente incorre devido ao fornecimento da RPO. No entanto, do ponto de vista prático, é mais conveniente distribuir a reserva entre todas as unidades da usina, com o intuito de otimizar o despacho e evitar expor as máquinas a rampas de carga maiores.

- Custos por perdas de eficiência na geração devido a mudanças nos pontos de operação programados: a disponibilidade de reserva deve levar em conta os períodos nos quais a regulação é feita "para cima", e os períodos nos quais a regulação é feita "para baixo". A reserva de regulação "para cima" ou, "up reserve", representa o aumento na geração, provocado por uma queda de frequência no sistema. Por outro lado, a reserva de regulação "para baixo" ou, "down reserve", representa a diminuição na geração, provocada por um aumento na frequência do sistema. 
Embora a regulação "para baixo" não representa o uso físico de capacidade adicional instalada, o ONS deve disponibilizar uma folga de geração negativa, por causa das restrições mínimas de produção das máquinas. Portanto, aparecem variações na geração programada que não aconteceriam caso a reserva não fosse fornecida.

Antes da operação em tempo real, o ONS determina o programa de geração das usinas hidrelétricas e termelétricas, o qual fica contido no Plano Diário de Produção - PDP (ONS, 2008a). No PDP, também são incluídas as restrições de reserva (montantes e alocação por agente). Como o montante de reserva de controle primário, secundário e terciário do sistema deve ser girante (ONS, 2008b), procura-se evitar a alocação da reserva em unidades geradoras desligadas. Assim, o ONS pode solicitar, além dos montantes de geração e de reserva, um número mínimo de unidades em operação. Se um agente gerador desconsidera as restrições de reserva, poderia programar as unidades dentro da usina de uma forma mais eficiente, cumprindo ainda com o programa de geração do PDP.

A diferença de perdas entre o ponto de operação que o gerador consegue, desconsiderando as restrições de reserva, em cada máquina $r$ pertencente à usina $i$, $p_{r-0}^{t}$, e o ponto programado que o gerador consegue considerando essas restrições, $p_{r}^{t}$, representa as perdas de eficiência. Aqui, supõe-se que o agente gerador tem liberdade para programar cada grupo de máquinas dentro da usina, desde que cumpra com o programa de geração. Assim, o custo por perdas de eficiência em cada período $t$ é determinado da seguinte forma:

$$
\begin{aligned}
c_{e f}^{\text {perdas }}= & \sum_{k}^{N(i, t)}\left\{p_{k-0}^{t}\left[\frac{1}{\eta_{k-0}}-1\right]-p_{k}^{t}\left[\frac{1}{\eta_{k}}-1\right]\right\} \\
& \times T_{t} \times c_{i}^{\text {perdas }}
\end{aligned}
$$

sendo:

$c_{i}^{\text {perdas }}$ : custo unitário das perdas na usina $i$ por $M W$ disponibilizado no período de tempo t em $\$ / M W / h^{4}$;

$p_{k-0}^{t}$ : geração atribuída para cada grupo gerador $k$ da usina $i$ no período $t$, pelo agente, sem considerar restrições de reserva impostas pelo ONS em $M W$;

$p_{k}^{t}$ : geração atribuída para cada grupo gerador $k$ da usina $i$ no período $t$, pelo agente, considerando as restrições de reserva impostas pelo ONS em $M W$;

\footnotetext{
${ }^{4} \mathrm{~A}$ unidade $\$ / M W / h$ é consistente com a unidade $\$ / M W h$, e é utilizada unicamente para evidenciar que corresponde a um custo de disponibilidade.
}

$\eta_{k-0}$ : eficiência de produção do grupo gerador $k$, quando a geração programada é $p_{k-0}^{t}$;

$\eta_{k}$ : eficiência de produção do grupo gerador $k$, quando a geração programada é $p_{k}^{t}$;

$T_{t}$ : duração do período $t$ em horas.

$N(i, t)$ : número de grupos geradores em operação na usina $i$ durante o período $t$.

Durante cada período $t$ programado, o agente pode estar submetido continuamente a perdas ou ganhos, dependendo do ponto de operação de cada unidade. Cabe determinar se, no final do horizonte de programação, esse componente realmente representa um custo para o gerador.

\subsubsection{Custo de uso}

O custo de uso representa o custo da energia entregue no fornecimento da reserva, e pode ser positivo ou negativo. Caso seja negativo, ele representa um custo de oportunidade que pode ser ressarcido em função da forma como a energia foi vendida uma vez que, dependendo do tipo de contrato do agente, essa parcela seria total ou parcialmente remunerada de forma implícita. O cálculo deste componente é ilustrado na equação (2).

$$
c_{u s o}=\left|P_{i-r}^{t}-P_{i}^{t}\right| \times T_{t} \times c_{\text {prod-i }}^{t}
$$

sendo:

$c_{\text {prod-i }}^{t}$ : custo variável de produção da usina $i$ no período $t$ em $\$ / M W h$;

$P_{i-r}^{t}$ : potência total gerada pela usina $i$ no período $t$ no despacho em tempo real em $M W$;

$P_{i}^{t}$ : potência total programada para a usina $i$ no período $t$ no PDP em $M W$.

Como a regulação primária atua de forma de forma contínua, "para cima" e "para baixo", e em pequenas quantidades, a parcela de energia devida ao uso da reserva para regulação "para cima", pode ser considerada compensada pela não geração, quando a regulação é "para baixo". Portanto, assume-se que a energia de fornecimento de reserva é devida principalmente à regulação secundária e terciária. Essa energia é controlada pelo ONS e deve ser compensada financeiramente ex-post. $\mathrm{Na}$ figura 2 são ilustradas as diferentes componentes da energia faturada.

$\mathrm{Na}$ figura 2, a energia de redespacho representa toda reprogramação da geração em tempo real destinada a outros usos, como o controle de tensão, a otimização do recurso hídrico, a otimização das perdas, etc. Adicionalmente, o 


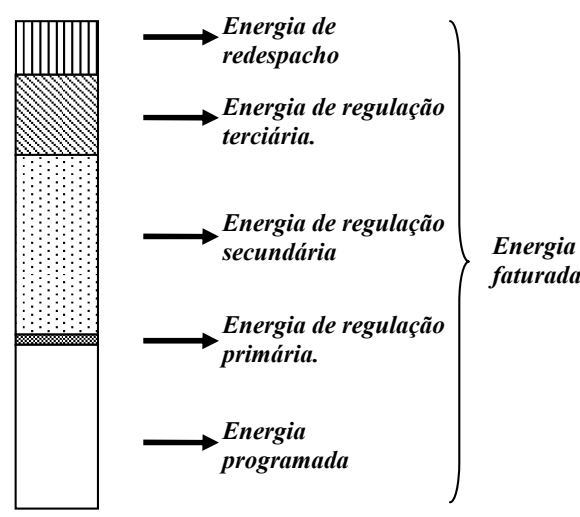

Figura 2: Componentes da energia faturada.

custo por frequência de uso é desconsiderado neste trabalho, assumindo que está embutido no próprio componente de custo de uso do serviço.

\subsubsection{Custo de oportunidade}

O custo de oportunidade se refere ao custo da energia não gerada ao disponibilizar determinada quantidade de reserva. Nos sistemas em que existe um mercado de energia de curto prazo (geralmente com um dia de antecedência), o custo de oportunidade é determinado em função do preço da energia resultante nesse mercado.

No caso do sistema brasileiro, a maior parte da energia é vendida em contratos de longo prazo. Além disto, o despacho é centralizado e baseado nas metas de planejamento de longo e médio prazo, razão pela qual, um gerador não pode modificar sua programação diária, visando gerar energia, no lugar de disponibilizar reserva. Portanto, o custo de oportunidade é afetado pelas decisões próprias do agente gerador, que determina os montantes de energia contratados, e pelas decisões do ONS, que determina os montantes de energia gerados.

No processo de comercialização, as diferenças entre os montantes de energia contratados e verificados entre os agentes, são liquidadas ao Preço de Liquidação das Diferenças - PLD, o qual é um valor calculado semanalmente, para cada patamar de carga, com base no Custo Marginal de Operação - CMO (CCEE, 2007a). Para o caso particular dos geradores hidrelétricos, os excedentes ou déficits com relação à energia contratada são valorados, em primeiro plano, num mecanismo de realocação de energia $\mathrm{MRE}^{5}$, ao preço da Tarifa de Energia de Otimização - TEO,

\footnotetext{
${ }^{5} \mathrm{O}$ MRE assegura que todas as usinas participantes recebam seus níveis de Energia Assegurada, desde que a geração total do MRE não esteja abaixo do total da Energia Assegurada do Sistema, independentemente de seus níveis reais de produção de energia (CCEE, 2007b).
}

e depois, se ainda existir alguma diferença, ao valor do PLD.

Como a disponibilidade e uso da reserva afeta as diferenças entre os montantes contratados e verificados, o custo de oportunidade pode ser calculado em função dessas diferenças, as quais, por sua vez, são função da TEO e do PLD.

O lucro de um agente gerador hidrelétrico $i$ pela venda de energia, é a diferença entre a receita liquida que recebe, $R E C_{i}$, e o custo de produção marginal próprio, $c_{\text {prod-i }}$. Se a receita, $R E C_{i}$, é dividida entre o montante de energia verificada, $E_{\text {ver }}$, obtém-se um preço equivalente da venda de energia $\pi_{E}$. Quando um gerador disponibiliza reserva, ele não incorre no custo de produção marginal próprio, já que a energia de reserva é fornecida unicamente quando o serviço é efetivamente utilizado em tempo real. O montante que o gerador poderia receber por essa reserva disponibilizada, $R_{i}$, seria $c_{\text {oport }}=\pi_{E} * R_{i}-c_{\text {prod }-i}$. Assim, quando a reserva for utilizada, o agente recebe adicionalmente o custo de produção $c_{\text {prod-i }}$. Em conjunto, o pagamento pela disponibilidade e uso da reserva (caso $100 \%$ dela fosse utilizada) seria $\pi_{E} * R_{i}-c_{\text {prod-i }}+c_{\text {prod-i }}$, recebendo, exatamente, o montante que ganharia caso a reserva tivesse sido vendida como energia gerada.

Resumindo, o custo de disponibilidade, considerando a perda de oportunidade, será:

$$
c_{\text {disp }}=c_{\text {oport }}+C M+c_{\text {perdas }}^{\text {ef }}+O C
$$

sendo:

$c_{\text {disp }}$ : custo de disponibilidade da usina;

$c_{\text {oport }}$ : custo de oportunidade da usina;

$C M$ : custo de manutenção da reserva;

$c_{\text {perdas }}^{\text {ef }}$ : custo pela perda de eficiência na usina devido ao fornecimento de reserva;

$O C$ : outros possíveis custos associados a processos administrativos.

Como o pagamento pela disponibilidade independe do uso, o OIS deve evitar requerimentos excessivos de reserva, assim como alocações arbitrárias, para não onerar este componente.

\section{MÉTODO PROPOSTO}

O modelo de despacho proposto possui as seguintes características:

- Mecanismo de competição: mercado diário; 
- Modelo de formação de preço: Market Clearing Price MCP;

- Estrutura do mercado: pseudocootimizada;

- Tipo de ofertas: ofertas semanais por disponibilidade para os serviços de reserva em $\$ / M W / h$ por parte dos geradores (demanda considerada fixa);

- Restrições de rede: consideradas no modelo;

- Tipo de produtos: reserva de regulação primária, secundária e terciária;

- Período de tempo de resolução do mercado: horário;

- Componentes de custos a serem ressarcidas: custos fixos, por disponibilidade e de uso;

- Gestor: o operador de rede (OIS ou ONS).

\subsection{Despacho centralizado}

O despacho de energia realizado no sistema brasileiro é centralizado e procura a otimização do recurso hídrico. O modelo está baseado na resolução dos programas NEWAVE e DECOMP (CCEE, 2007a), os quais resolvem um problema de despacho hidrotérmico com diferentes escalas de tempo. Após a resolução destes modelos, fica determinado o PLD, o qual é calculado para cada patamar de carga e para cada região. Atualmente, este preço permanece constante durante toda a semana. Assim, durante a operação diária, o ONS realiza o despacho das unidades visando atingir as metas semanais.

No Brasil, a maior porcentagem da produção é hidrelétrica. Assumindo a geração térmica distribuída uniformemente ao longo da semana, de forma a atingir as metas semanais, considera-se que as variações da demanda são cobertas apenas por usinas hidrelétricas (Arce, 2006). Com esta abordagem, é possível realizar um despacho unicamente hidrelétrico com a cadeia de programação diária ilustrada na figura 3.

De acordo com essa figura, a programação diária segue as diretrizes do planejamento de longo e médio prazo. Para a operação de curto prazo, existe a necessidade de realizar o despacho da geração cumprindo com certas restrições que podem ser classificadas da seguinte maneira:

- Restrições próprias da geração. Neste grupo são incluídos os limites das máquinas geradoras, o atendimento das metas planejadas, o número máximo de máquinas em operação, etc;

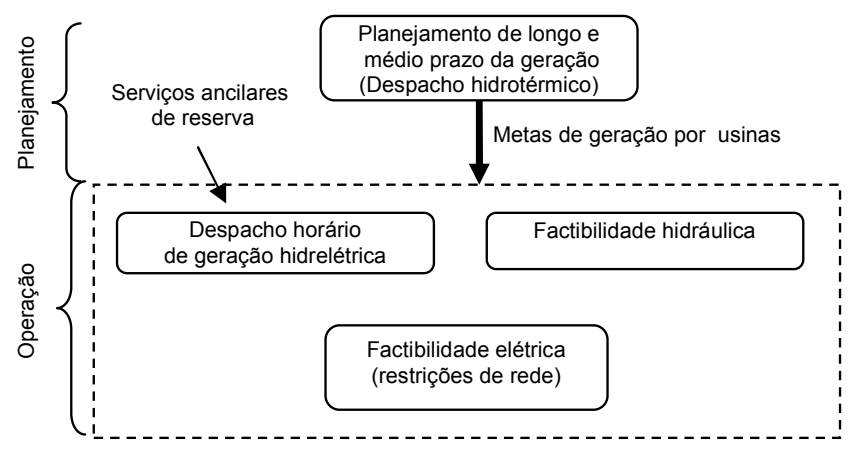

Figura 3: Cadeia de programação do despacho de geração.

- Restrições hidráulicas. Neste grupo são consideradas as restrições de armazenamento nos reservatórios, os níveis de defluência máximo e mínimo, etc;

- Restrições de transmissão. Correspondem aos limites de tensão, estabilidade, equações de balanço nodal, etc.

Os serviços ancilares de reserva operativa são considerados restrições próprias da geração, e fazem parte do despacho de geração hidrelétrico.

\subsection{Definição da estrutura do mercado}

A escolha de uma estrutura pseudocootimizada foi realizada a partir da consideração do conjunto de alternativas descrito a seguir:

1. Reserva de cada gerador fixada pelo ONS no despacho programado;

2. Reserva de cada gerador incluída no despacho programado como uma variável, assumindo custos iguais para todos os agentes;

3. Reserva de cada gerador incluída no despacho programado como uma variável, assumindo um preço de oferta por parte de cada gerador (pseudocootimização);

4. Reserva de cada gerador não considerada no despacho programado, e determinada mediante um leilão separado com ofertas tipo preço-quantidade por parte de cada gerador (otimização sequencial);

As duas primeiras opções correspondem a um cenário de não mercado. As duas últimas correspondem às alternativas pseudocootimizada e sequencial. No esquema sequencial, primeiro é realizado o despacho de geração e depois é realizado o despacho de reserva. Como resultado da 
otimização, pode acontecer que a reserva alocada no sistema não seja estritamente girante, já que essa restrição não foi considerada no despacho de geração inicial. Isto levaria à necessidade de ligar mais máquinas e modificar o despacho previamente realizado, tornando o processo ineficiente. Portanto, seleciona-se o esquema que neste trabalho é chamado de pseudocootimizado. Enquanto o esquema cootimizado otimiza o despacho de energia e reserva, no esquema pseudocootimizado, é otimizado unicamente o despacho de potência e reserva, uma vez que a energia já foi otimizada no planejamento de longo e médio prazo. Caso no sistema brasileiro fosse implementado um modelo de despacho hidrotérmico horário, o esquema anterior considerando esse modelo seria um esquema de cootimização.

\subsection{Tipo de oferta}

Em geral, as ofertas podem ter diversos formatos, dependendo da forma como o mercado é desenhado.

1. Ofertas simples por capacidade de reserva:

(a) Ofertas de preço de disponibilidade em $\$ / M W / h$;

(b) Ofertas que indicam o preço de disponibilidade em $\$ / M W / h$ e a capacidade de reserva em $M W$.

2. Ofertas duplas por capacidade e energia de reserva:

(a) Ofertas de preço de disponibilidade e preço de uso da energia de reserva em $\$ / M W / h$ e $\$ / M W h$;

(b) Ofertas de preço de disponibilidade-uso e capacidade de reserva disponibilizada em $\$ / M W / h-\$ / M W h$ e $M W$.

Uma comparação das ofertas simples e duplas pode ser encontrada em (Shahidehpour et al., 2002), (Swider, 2007) e (Stoft, 2002). As ofertas duplas representam um esquema de ofertas mais complexo, que inclui uma oferta pelo uso da reserva, no caso em que o serviço é utilizado em tempo real. Nesta situação, o agente gerador deve ter uma previsão da demanda de energia de reserva para calcular o valor da oferta de uso. Por outro lado, a quantidade de reserva é especificada quando o gerador participa de um mercado de reserva sequencial, no qual se deve indicar a capacidade disponível para o(s) mercado(s) de reserva e o mercado de energia. No caso de um mercado cootimizado, unicamente se indica a capacidade máxima da unidade geradora e eventuais restrições de rampa, sendo a reserva determinada de forma ótima pelo algoritmo de despacho.

Nas ofertas simples, os geradores oferecem um preço pela disponibilidade, sendo o uso do serviço remunerado ex-post, baseado no custo de produção, $c_{\text {prod }}$. Neste trabalho será considerada a oferta simples tipo a, já que o operador de rede realiza o despacho centralizado e pode alocar a energia e a reserva simultaneamente.

No Brasil, como o PLD só muda semanalmente, os geradores podem manter a oferta por disponibilidade constante ao longo da semana, embora a atribuição e determinação do preço devam ser realizada em forma horária, em função dos requerimentos operativos. De acordo com o ilustrado na seção 3.1.4, as ofertas por disponibilidade poderiam ter um preço máximo igual ao PLD, já que este é o valor mais alto ao qual as diferenças de energia são valoradas. $\mathrm{O}$ valor das ofertas depende da análise que cada agente gerador faz de seus custos de disponibilidade associados (equação (3)) e de sua estratégia de jogo no mercado. No modelo matemático apresentado na seguinte seção, o valor dessas ofertas é assumido conhecido, e representa um dado de entrada no algoritmo de despacho.

Por outro lado, os custos fixos podem ser ressarcidos de forma separada ao processo de otimização, uma vez que não dependem da operação do sistema, enquanto o pagamento dos custos de uso seria realizado após a operação, em função dos dados de energia de reserva registrada (figura 2) e do custo de produção $c_{\text {prod }}$.

\subsection{Modelagem matemática}

O modelo de despacho hidrelétrico considerado neste trabalho (equações (4) a (12)) é uma versão modificada do modelo apresentado em (Arce, 2006). A função de perdas daquele trabalho foi desconsiderada para conservar a linearidade da função objetivo. Assim, o despacho proposto é um modelo centralizado no qual, o ONS, além de programar a potência a ser gerada em cada usina, pode programar o número de máquinas em operação, visando garantir o requerimento de reserva girante do sistema. Para simplificar a modelagem, foi considerado que todas as máquinas que pertencem a uma mesma usina possuem características iguais. 


$$
\begin{aligned}
& \operatorname{Min} \sum_{t}^{T} \sum_{i}^{N} c_{i}^{p / p} \times\left|n_{i}^{t}-n_{i}^{t-1}\right| \\
& \quad+\sum_{t}^{T} \sum_{i}^{N} \pi_{i}^{t} \times R_{i-u p}^{t} \\
& \text { s.a. } \\
& \sum_{i}^{N} P_{i}^{t}=D^{t} \forall t \\
& \sum_{t}^{T} P_{i}^{t}=T \times m_{i} \forall i \\
& \sum_{i}^{N} R_{i-\text { up }}^{t}=R_{\text {req }}^{u p} \forall t \\
& \sum_{i}^{N} R_{i-\text { do }}^{t}=R_{\text {req }}^{\text {do }} \forall t \\
& P_{i}^{t}+R_{i-u p}^{t} \leq p_{i}^{\text {max }} \times n_{i}^{t} \forall i, t \\
& P_{i}^{t}-R_{i-\text { do }}^{t} \geq p_{i}^{\text {min }} \times n_{i}^{t} \forall i, t
\end{aligned}
$$

$$
\begin{aligned}
& n_{i}^{\text {min }} \leq n_{i}^{t} \leq n_{i}^{\max } \forall i, t \\
& n_{i}^{t} \in \aleph \forall i, t
\end{aligned}
$$

sendo:

$T$ : horizonte de tempo considerado;

$N$ : número de usinas hidrelétricas;

$c_{i}^{p / p}:$ custos de partida/parada da unidade geradora da usina $i$ em $[\$]$;

$\pi_{i}$ : preço da oferta de disponibilidade de reserva de regulação "para cima" da usina $i$, durante a semana, em $\$ / M W / h$;

$n_{i}^{t}$ : número de máquinas em operação da usina $i$ no período $t$

$P_{i}^{t}$ : potência total programada na usina $i$ no período $t$ em $[M W]$;

$D^{t}$ : demanda do sistema descontada da geração térmica durante o período $t$ em $[M W]$;

$m_{i}$ : meta energética média da usina hidrelétrica $i$ em [MWmédio];

$R_{i-u p}^{t}:$ reserva total de regulação "para cima" da usina $i$ no período $t$ em $M W$;
$R_{i-d o}^{t}:$ reserva total de regulação "para baixo" da usina $i$ no período $t$ em $M W$;

$p_{i}^{\max }:$ geração máxima de cada grupo gerador da usina $i$ em $M W$;

$p_{i}^{\text {min }}$ : geração mínima de cada grupo gerador da usina $i$ em $M W$;

$R_{u p}^{t}$ : demanda de reserva de regulação "para cima" do sistema no período $t$ em $M W$;

$R_{d o}^{t}$ : demanda de reserva de regulação "para baixo" do sistema no período $t$ em $M W$;

ふ: conjunto dos números naturais.

A função objetivo, equação (4), minimiza o número de partidas e paradas das máquinas do sistema, assim como o custo de aquisição da reserva de regulação "para cima". A equação (5) representa o balanço de potência ativa, enquanto, a equação (6), representa as metas de geração de cada usina. As restrições de demanda de reserva de regulação "para cima" e "para baixo" são consideradas nas equações (7) a (10). Neste ponto, cabe destacar que a quantidade e flexibilidade das máquinas hidrelétricas, existentes no sistema brasileiro, tornam difícil o atendimento dos limites de geração mínima das unidades. Assim, o ONS só consideraria os requerimentos positivos de reserva, isto é, a reserva de regulação "para cima". No modelo aqui apresentado, a reserva de regulação "para abaixo" foi conservada para evitar a perda de generalidade da modelagem. Finalmente, a equação (11) estabelece os limites para o número possível de máquinas em operação dentro de cada usina e, a equação (12), indica que $n_{i}^{t}$ é uma variável inteira e positiva.

Pode-se observar que na função objetivo não foram consideradas as ofertas por disponibilidade de reserva de regulação "para baixo". Neste caso, foi assumido que a disponibilidade dessa reserva não representa realmente um custo para o agente, já que isto leva a um aumento nos níveis de geração dos grupos geradores, com a finalidade de evitar a operação em zonas proibidas por restrições de geração mínima. O fato de que um agente incremente sua geração, por este aspecto, é interpretado como um ganho indireto pela venda de energia. Além disso, deve ser notado que a reserva, $R_{i-u p}^{t}$, representa o montante total de reserva de regulação "para cima", isto é, a soma das reservas de regulação primária, secundária e terciária, em cada gerador da usina $i$. O mesmo raciocínio se aplica para a reserva de regulação "para baixo", $R_{i-d o}^{t}$. A simplificação anterior se realiza porque a proposta considera usinas com a mesma tecnologia de geração (hidrelétricas) e toda a reserva, incluindo a reserva de regulação terciária, é assumida de 
natureza girante (ONS, 2008b).

Os termos do problema descrito anteriormente são lineares, excetuando o valor absoluto da função objetivo, que descreve o número de partidas e paradas das máquinas em cada período. Mesmo assim, o problema pode ser manipulado e formulado como um problema de programação linear inteiro-misto.

\subsection{Restrições hidráulicas}

Para considerar as restrições hidráulicas no despacho do dia seguinte, assume-se que a queda líquida do reservatório não varia, de forma significativa, ao longo do horizonte de programação (ex. um dia). Sendo a queda hidráulica um valor conhecido e constante, pode ser realizada uma simplificação das restrições apresentadas em (Cicogna, 1999). Assim, as restrições hidráulicas do modelo proposto são as seguintes:

$$
\begin{aligned}
& x_{i}^{t}=x_{i}^{t-1}+\left[y_{i}^{t}+\sum_{k \in \Omega_{i}} u_{k}^{t}-u_{i}^{t}\right] \times \frac{\Delta t^{t}}{10^{6}} \\
& P_{i}^{t}=k_{e q} \times \eta_{i}^{t} \times \hat{h}_{l i}^{t} \times q_{i}^{t} \\
& u_{i}^{t}=q_{i}^{t}+v_{i}^{t} \\
& x_{i}^{\text {min }} \leq x_{i}^{t} \leq x_{i}^{\max } \\
& u_{i}^{\text {min }} \leq u_{i}^{t} \leq u_{i}^{\max } \\
& v_{i}^{t} \geq 0
\end{aligned}
$$

sendo:

$x_{i}^{t}$ : volume do reservatório da usina $i$ no final do período $t$ em $\left[\mathrm{Hm}^{3}\right]$;

$u_{i}^{t}$ : vazão defluente da usina $i$ durante o período $t$ em $\left[\mathrm{m}^{3} / \mathrm{s}\right]$;

$q_{i}^{t}$ : vazão turbinada pela usina $i$ durante o período $t$ em $\left[\mathrm{m}^{3} / \mathrm{s}\right]$;

$v_{i}^{t}$ : vazão vertida pela usina $i$ durante o período $t$ em $\left[\mathrm{m}^{3} / \mathrm{s}\right]$;

$y_{i}^{t}$ : vazão incremental afluente à usina $i$ durante o período $t$ em $\left[m^{3} / s\right]$;

$\Delta t^{t}$ : duração de cada período de tempo $t$ em $[s]($ ex. $3600 s)$;

$\Omega_{i}$ : conjunto das usinas imediatamente a montante da usina $i$

$k_{e q}$ : constante que integra o efeito da gravidade e o peso específico da água, $k=9,81 \times 10^{-3}\left[\frac{M W}{\left(m^{3} / s\right) \cdot m}\right]$;

$\eta_{i}^{t}$ : eficiência equivalente dos conjuntos turbina-gerador da usina $i$ no período $t$;

$\hat{h}_{l i}^{t}$ : queda líquida da usina $i$ durante o período $t$ em $[m]$ (valor conhecido);

A equação (13) representa a restrição de balanço hidráulico, enquanto a equação (14) mostra a restrição de produção hidráulica da usina. Na equação (15) se relaciona a defluência em função do vertimento e a vazão turbinada. Nas equações (16) a (18) são estabelecidos os limites mínimo e máximo de volume armazenado, a vazão defluente e a vazão vertida respectivamente.

\subsection{Restrições elétricas}

As restrições elétricas geralmente são incorporadas usando uma modelagem AC ou CC. A modelagem AC permite considerar as perdas e o fluxo de potência reativa, no entanto, possui característica não linear. Por outro lado, o modelo CC possui característica linear, mas não considera aqueles aspectos. Para manter a simplicidade e consistência das restrições elétricas com o despacho hidrelétrico e as restrições hidráulicas apresentadas, a rede será representada através do modelo CC (Silva et al., 2005).

$$
\begin{aligned}
& \sum_{s \in \Psi_{r}} f_{r s}^{t}+g_{r}^{t}=d_{r}^{t} \\
& f_{r s}^{t}-\gamma_{r s} \times n_{r s}^{0} \times\left(\theta_{r}^{t}-\theta_{s}^{t}\right)=0 \\
& \left|f_{r s}\right| \leq n_{r s}^{0} \times \overline{f_{r s}}
\end{aligned}
$$

Nas equações anteriores, $\gamma_{r s}$ representa a susceptância da linha $r-s$ em [p.u], $n_{r s}^{0}$ o número de circuitos existentes no trecho $r-s, f_{r s}^{t}$ o fluxo de potência no trecho $r-s$ no período $t$ em [p.u.], $\theta_{r}^{t}$ a abertura angular da barra $r$ no período $t$ em [rad.], $g_{r}^{t}$ a geração na barra $i$ no período $t$ em [p.u.]. A geração está representada pelo conjunto de usinas $\Phi_{i}$ conectadas ao nó $r, g_{r}^{t}=\sum_{\Phi_{i}} P_{i}^{t} / P_{B A S E}$. A potência base do sistema é representada por $P_{B A S E}$. A variável $d_{r}^{t}$, representa a demanda na barra $r$ no período $t$ em [p.u.]. A demanda uninodal, que se mostra na equação (5), é a soma das demandas em todas as barras do sistema, as quais devem ser corrigidas ao descontar a porcentagem que é suprida por geração térmica, $D^{t}=\sum_{r} d_{r}^{t}$. $P_{B A S E}$. Finalmente $\Psi_{r}$ representa o conjunto de linhas conectadas ao nó $r$.

A equação (19) representa o balanço de potência em cada nó do sistema e, a equação (20), relaciona o fluxo pelas linhas em função das aberturas angulares. Na equação (21) se estabelecem os limites de fluxo de potência pelas linhas. 


\subsection{Adaptação do modelo de despacho global}

O problema de programação da geração para o dia seguinte, deve encontrar as unidades geradoras necessárias para atender a demanda horária ao mínimo custo e cumprindo todas as restrições operacionais. Assim, a formulação do despacho global está dada pelo seguinte problema de programação linear inteira mista ${ }^{6}$ :

$$
\begin{aligned}
& \operatorname{Min} \quad \sum_{t}^{T} \sum_{i}^{N} c_{i}^{p / p} \times\left|n_{i}^{t}-n_{i}^{t-1}\right| \\
& +\sum_{t}^{T} \sum_{i}^{N}\left[\pi_{i}^{t} \times R_{i-u p}^{t}\right] \\
& \sum_{t}^{T} P_{i}^{t}=T \times m_{i} \forall i \\
& \sum_{i}^{N} R_{i-u p}^{t}=R_{r e q}^{u p} \forall t \\
& \sum_{i}^{N} R_{i-d o}^{t}=R_{r e q}^{d o} \forall t \\
& P_{i}^{t}+R_{i-u p}^{t} \leq p_{i}^{\max } \times n_{i}^{t} \forall i, t \\
& P_{i}^{t}-R_{i-d o}^{t} \geq p_{i}^{\text {min }} \times n_{i}^{t} \forall i, t \\
& n_{i}^{\min } \leq n_{i}^{t} \leq n_{i}^{\max } \forall i, t \\
& n_{i}^{t} \in \aleph \forall i, t \\
& x_{i}^{t}=x_{i}^{t-1}+\left[y_{i}^{t}+\sum_{k \in \Omega_{i}} u_{k}^{t}-u_{i}^{t}\right] \cdot \frac{\Delta t^{t}}{10^{6}} \\
& P_{i}^{t}=k_{e q} \times \eta_{i}^{t} \times \hat{h}_{l i}^{t} \times q_{i}^{t} \\
& u_{i}^{t}=q_{i}^{t}+v_{i}^{t} \\
& x_{i}^{\text {min }} \leq x_{i}^{t} \leq x_{i}^{\max } \\
& u_{i}^{\text {min }} \leq u_{i}^{t} \leq u_{i}^{\max } \\
& v_{i}^{t} \geq 0 \\
& d_{r}^{t}=\sum_{s \in \Psi_{r}} f_{r s}^{t}+\sum_{\Phi_{i}} P_{i}^{t} / P_{B A S E} \\
& f_{r s}^{t}-\gamma_{r s} \times n_{r s}^{0} \times\left(\theta_{r}^{t}-\theta_{s}^{t}\right)=0 \\
& \left|f_{r s}\right| \leq n_{r s}^{0} \times \overline{f_{r s}}
\end{aligned}
$$

Pode-se observar que a restrição de balanço de potência

\footnotetext{
${ }^{6} \mathrm{Da}$ mesma forma que o modelo descrito pelas equações equações (4) a (12), este modelo também pode ser manipulado e formulado como um problema de programação linear inteira mista.
}

uninodal (equação (5)) foi retirada, pois está implícita na restrição de balanço de potência nodal (equação (36)).

\section{TESTES E RESULTADOS}

O seguinte exemplo ilustra o despacho de potência e reserva para três períodos de programação. Consideram-se três usinas hidrelétricas (duas com reservatório e uma a fio d'água) dispostas como ilustrado na figura 4. As características de cada usina se mostram nas tabelas 1 e 2. Na tabela 1, os valores de potência mínima, máxima e custos de partida e parada são especificados por unidade geradora. A eficiência, $\eta_{i}^{t}$, é assumida constante e igual a $0,9^{7}$. Também foi assumido que cada usina funciona inicialmente com 3 unidades geradoras e foi utilizada uma potência base de $100 \mathrm{MW}$.

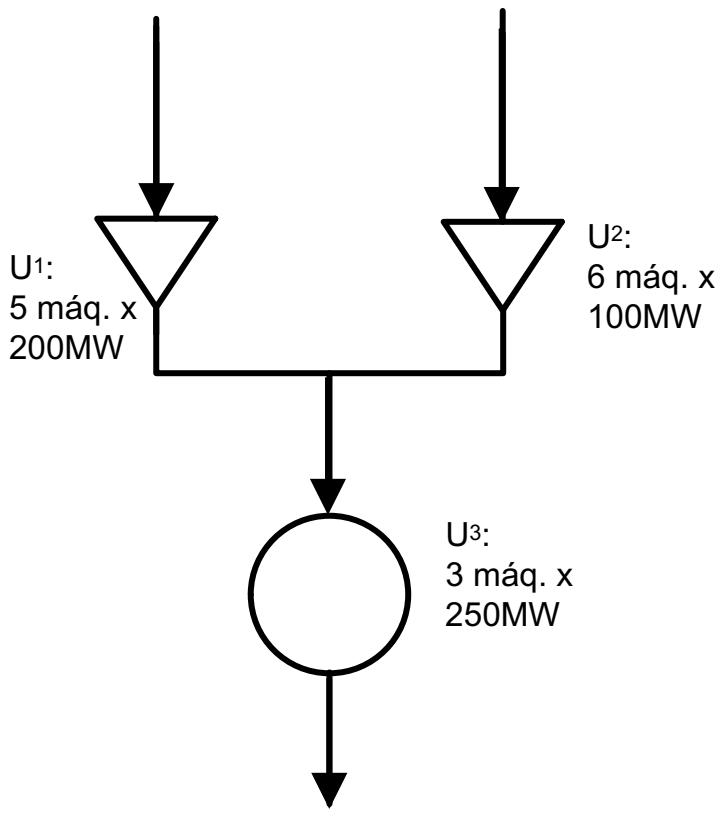

Figura 4: Configuração hidráulica do sistema.

Tabela 1: Dados dos geradores.

\begin{tabular}{lccc}
\hline & $G_{1}$ & $G_{2}$ & $G_{3}$ \\
\hline$p_{i}^{\min }[M W]$ & 80 & 40 & 100 \\
$p_{i}^{\max }[M W]$ & 200 & 100 & 250 \\
$n_{i}^{\min }$ & 0 & 0 & 0 \\
$n_{i}^{\max }$ & 5 & 6 & 3 \\
$n_{i}^{0}$ & 3 & 3 & 3 \\
$c_{i}^{p / p}[\$]$ & 5 & 4 & 3 \\
$m_{i}[M W$ med $]$ & 600 & 200 & 600 \\
\hline
\end{tabular}

${ }^{7}$ Para valores de $P_{i}^{t}$ e $\hat{h}_{l i}^{t}$ conhecidos, também é possível aproximar o valor da efi ciência a partir das curvas colina dos geradores. 
Tabela 2: Dados dos reservatórios

\begin{tabular}{lccc}
\hline & $G_{1}$ & $G_{2}$ & $G_{3}$ \\
\hline$x_{i}^{\text {min }}\left[\mathrm{Hm}^{3}\right]$ & 1347 & 1143 & 2811 \\
$x_{i}^{\text {max }}\left[\mathrm{Hm}^{3}\right]$ & 1412 & 3000 & 2811 \\
$u_{i}^{\text {min }}\left[\mathrm{m}^{3} / \mathrm{s}\right]$ & 100 & 60 & 40 \\
$u_{i}^{\text {max }}\left[\mathrm{m}^{3} / \mathrm{s}\right]$ & 1000 & 600 & 1400 \\
$x_{i}^{0}\left[\mathrm{Hm}^{3}\right]$ & 1412 & 1143 & 2811 \\
$\hat{h}_{l i}[\mathrm{~m}]$ & 175 & 150 & 100 \\
\hline
\end{tabular}

A tabela 3 mostra as afluências previstas em cada bacia para cada período de tempo, as ofertas de disponibilidade de reserva de regulação "para cimat't' e a demanda de energia e de reserva do sistema. Com fins ilustrativos, foram simuladas ofertas de disponibilidade diferentes em cada período. No contexto brasileiro, essas ofertas permaneceriam constantes ao longo da semana, de acordo com a discussão apresentada na seção (4.3). A tabela 4 e a figura 5 mostram os dados e a configuração da rede respectivamente.

Tabela 3: Afluências, preços e demandas previstas.

\begin{tabular}{lccc}
\hline & $T_{1}$ & $T_{2}$ & $T_{3}$ \\
\hline$y_{1}\left[\mathrm{~m}^{3} / \mathrm{s}\right]$ & 1000 & 900 & 600 \\
$y_{2}\left[\mathrm{~m}^{3} / \mathrm{s}\right]$ & 600 & 900 & 1200 \\
$y_{3}\left[\mathrm{~m}^{3} / \mathrm{s}\right]$ & 0 & 0 & 0 \\
$\pi_{1}[\$ / M W / h]$ & 98 & 14 & 42 \\
$\pi_{2}[\$ / M W / h]$ & 87 & 49 & 57 \\
$\pi_{3}[\$ / M W / h]$ & 82 & 32 & 10 \\
$D[M W]$ & 2000 & 1500 & 700 \\
$R^{\text {up }}[M W]$ & 200 & 150 & 100 \\
$R^{\text {down }}[M W]$ & 200 & 150 & 100 \\
\hline
\end{tabular}

Tabela 4: Dados das linhas.

\begin{tabular}{cccc}
\hline Linha & $x_{i j}[p . u]$. & $N c$ & $f_{i j}^{\max } /$ cto. $[M W]$ \\
\hline$L_{1-2}$ & 0,0103 & 1 & 600 \\
$L_{1-3}$ & 0,0414 & 1 & 600 \\
$L_{1-4}$ & 0,0329 & 2 & 600 \\
$L_{2-3}$ & 0,0423 & 1 & 600 \\
$L_{3-4}$ & 0,0618 & 2 & 600 \\
\hline
\end{tabular}

$\mathrm{Na}$ solução do despacho global foi utilizado o solver comercial CPLEX 8.1 sob a interface GAMS. Com o objetivo de comparar a alocação da reserva em diferentes situações, foram simulados os cenários ilustrados na seção 4.2, excetuando o cenário de otimização sequencial (o qual se encontra fora do escopo deste trabalho):

- Cenário 1: a reserva não possui um custo de disponibilidade e é atribuída proporcionalmente à capacidade instalada das usinas. Os porcentagens de atribuição sobre a demanda de reserva do sistema são:

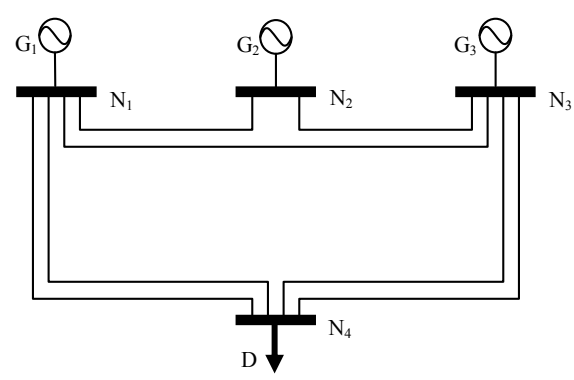

Figura 5: Configuração elétrica do sistema.

Tabela 5: Alocação da reserva em cada cenário [MW].

\begin{tabular}{clccc}
\hline & & $T_{1}$ & $T_{2}$ & $T_{3}$ \\
\hline \multirow{4}{*}{ Cenário 1 } & $R_{1-u p}$ & 84 & 63 & 42 \\
& $R_{2-u p}$ & 51 & 38,25 & 25,50 \\
& $R_{3-u p}$ & 65 & 48,75 & 32,50 \\
\hline \hline \multirow{3}{*}{ Cenário 2 } & $R_{1-u p}$ & 0 & 40 & 100 \\
& $R_{2-u p}$ & 200 & 110 & 0 \\
& $R_{3-u p}$ & 0 & 0 & 0 \\
\hline \hline \multirow{3}{*}{ Cenário 3 } & $R_{1-u p}$ & 0 & 150 & 0 \\
& $R_{2-u p}$ & 0 & 0 & 0 \\
& $R_{3-u p}$ & 200 & 0 & 100 \\
\hline
\end{tabular}

$42 \%$ para a usina $1,25,5 \%$ para a usina 2 e $32,5 \%$ para a usina 3 .

- Cenário 2: todos os geradores possuem uma oferta de disponibilidade de reserva igual a $14 \$ / M W h$, enquanto o montante de reserva que é atribuído a casa usina é uma variável determinada pelo despacho programado.

- Cenário 3: a reserva é uma variável determinada pelo despacho programado, o qual considera as ofertas ilustradas na tabela 3 (pseudocootimização).

As tabelas 5 e 6 mostram a alocação da reserva "para cima" e o número de unidades em operação para cada cenário.

Pode-se notar, nessas tabelas, que no cenário 1 , a reserva é distribuída entre todos os geradores do sistema, sendo que a usina 1 apresenta 5 partidas/paradas das unidades, enquanto a usina 2 apresenta 6 . Como o custo de disponibilidade não é levado em conta no despacho, a reserva é distribuída entre todos os geradores habilitados para o fornecimento.

No cenário 2, a reserva foi alocada nas usinas 1 e 2 , e o número de partidas/paradas resultou menor do que no cenário 1. Neste caso, como a oferta de disponibilidade é igual para todas as usinas, o despacho não aloca a reserva visando minimizar o preço de disponibilidade, e sim o número de partidas/paradas das unidades. 
Tabela 6: Número de unidades em operação em cada cenário.

\begin{tabular}{cccccc}
\hline & $T_{0}$ & $T_{1}$ & $T_{2}$ & $T_{3}$ & No. $\mathrm{p} / \mathrm{p}$ \\
\hline$n_{1}$ & 3 & 5 & 5 & 2 & 5 \\
$n_{2}$ & 3 & 5 & 2 & 1 & 6 \\
$n_{3}$ & 3 & 3 & 3 & 3 & 0 \\
\hline \hline$n_{1}$ & 3 & 5 & 3 & 3 & 4 \\
$n_{2}$ & 3 & 5 & 4 & 4 & 3 \\
$n_{3}$ & 3 & 3 & 3 & 2 & 1 \\
\hline \hline$n_{1}$ & 3 & 5 & 5 & 2 & 5 \\
$n_{2}$ & 3 & 5 & 1 & 1 & 6 \\
$n_{3}$ & 3 & 3 & 3 & 3 & 0 \\
\hline
\end{tabular}

Na método proposto neste trabalho, e que corresponde ao cenário 3, a reserva foi alocada nas usinas 1 e 3 , que são justamente as usinas com menores preços de oferta nos períodos considerados. Esses preços resultaram em 82,14 e $10 \$ / M W / h$ para os períodos $T_{1}, T_{2}$ e $T_{3}$ respectivamente. $\mathrm{O}$ número de partidas/paradas resultou maior do que no cenário 2 , já que a disponibilidade de reserva apresenta um peso maior na função objetivo do que as partidas/paradas das unidades. A consideração do número de unidades em operação é importante, já que se evita o chaveamento desnecessário de unidades, possíveis sobrecustos de operação para os agentes e o consequente incremento nas ofertas de disponibilidade.

As tabelas 7 e 8 apresentam outros resultados do despacho programado no cenário proposto. Esses resultados mostram o plano de produção que cumpre com as metas de geração, assim como os pontos de operação das redes elétrica e hidráulica.

Do ponto de vista do custo da reserva, no cenário 1 , o custo do serviço é desconsiderado, dado que a alocação da reserva obedece unicamente um critério técnico. No cenário 2 , o custo do serviço é considerado, mas a alocação da reserva continua obedecendo unicamente um critério técnico, já que o custo é igual para todos os agentes. No cenário 3, os agentes são livres para ofertar um preço que lhes permita recuperar seus custos de disponibilidade, e o operador pode alocar a reserva de forma eficiente utilizando critérios técnicos e econômicos.

\section{CONCLUSÕES}

$\mathrm{Na}$ primeira parte deste trabalho foram descritos alguns aspectos da reserva operativa, destacando, os componentes de custo associados ao fornecimento deste serviço. Nessa descrição foi ilustrado que o custo de oportunidade pode ser incorporado no custo de disponibilidade, o qual constitui a base para realizar as ofertas no cenário de mercado. Por
Tabela 7: Valores do despacho programado.

\begin{tabular}{lccc}
\hline & $T_{1}$ & $T_{2}$ & $T_{3}$ \\
\hline$P_{1}[M W]$ & 990 & 650 & 160 \\
$P_{2}[M W]$ & 460 & 100 & 40 \\
$P_{3}[M W]$ & 550 & 750 & 500 \\
$R_{1}^{\text {down }}[M W]$ & 200 & 0 & 0 \\
$R_{2}^{\text {down }}[M W]$ & 0 & 0 & 0 \\
$R_{3}^{\text {down }}[M W]$ & 0 & 150 & 100 \\
$u_{1}\left[\mathrm{~m}^{3} / \mathrm{s}\right]$ & 1000 & 1000 & 506,3 \\
$u_{2}\left[\mathrm{~m}^{3} / \mathrm{s}\right]$ & 400 & 400 & 60 \\
$u_{3}\left[\mathrm{~m}^{3} / \mathrm{s}\right]$ & 1400 & 1400 & 566,3 \\
$v_{1}\left[\mathrm{~m}^{3} / \mathrm{s}\right]$ & 359,3 & 579,3 & 402,8 \\
$v_{2}\left[\mathrm{~m}^{3} / \mathrm{s}\right]$ & 52,7 & 324,5 & 29,8 \\
$v_{3}\left[\mathrm{~m}^{3} / \mathrm{s}\right]$ & 777,1 & 550,5 & 0 \\
\hline
\end{tabular}

Tabela 8: Fluxos e ângulos do sistema.

\begin{tabular}{cccc}
\hline Linha & $T_{1}$ & $T_{2}$ & $T_{3}$ \\
\hline$L_{1-2}[M W]$ & $-166,7$ & $-35,6$ & $-63,2$ \\
$L_{1-3}[M W]$ & 191,6 & 42,4 & $-34,1$ \\
$L_{1-4}[M W]$ & 965,1 & 643,2 & 257,3 \\
$L_{2-3}[M W]$ & 293,3 & 64,4 & $-23,2$ \\
$L_{3-4}[M W]$ & 1034,9 & 856,8 & 442,7 \\
\hline$\theta_{1}[$ rad. $]$ & 0 & 0 & 0 \\
$\theta_{2}[$ rad. $]$ & 0,017 & 0,004 & 0,007 \\
$\theta_{3}[$ rad. $]$ & $-0,079$ & $-0,018$ & 0,014 \\
$\theta_{4}[$ rad. $]$ & $-0,298$ & $-0,199$ & $-0,080$ \\
\hline
\end{tabular}

outro lado, sugeriu-se que o custo de uso pode ser ressarcido financeiramente ex-post, em função do montante de energia de reserva fornecida. $\mathrm{Na}$ segunda parte deste artigo, foi apresentada uma alternativa de valorização da reserva através de um mercado. A proposta foi construída utilizando uma estrutura de mercado pseudocootimizada com ofertas simples, indicando, unicamente, o preço de disponibilidade do serviço. Posteriormente, foi apresentado um modelo de despacho hidrelétrico, baseado na modelagem matemática de (Arce, 2006), e, incorporando, adicionalmente, as restrições hidráulicas descritas em (Cicogna, 1999), assim como as restrições elétricas do modelo clássico CC linearizado. Essas restrições foram integradas de forma consistente em um modelo denominado de despacho global. O modelo permite, além de atribuir e valorizar a reserva, representar o efeito do acoplamento em cascata e da interconexão elétrica entre as usinas. A proposta foi avaliada através de um sistema teste, sendo que os resultados mostram a alocação eficiente da reserva, em função da oferta de menor preço, assim como a factibilidade e otimalidade operativa. Além disso, o método pode ser estendido para considerar um número maior de usinas interconectadas. 


\section{AGRADECIMENTOS}

À Coordenação e Aperfeiçoamento de Pessoal de Nível Superior - CAPES e à Fundação de Ensino, Pesquisa e Extensão de Ilha Solteira - FEPISA pelo suporte financeiro.

\section{REFERÊNCIAS}

Agurto, G. M. (2004). Um Modelo de Despacho Econômico para Reservas Operativas, Dissertação de mestrado, Universidade Estadual de Campinas, Facultade de Engenharia Elétrica e Computação, Campinas, Brasil.

ANNEL (2003). Resolução no. 265, de 10 de junho de 2003, Brasilia.

URL: http://www.aneel.gov.br/

ANNEL (2007). Resolução no 251, de 13 de fevereiro de 2007, Brasília.

URL: http: //www. aneel.gov.br/

Arce, A. S. (2006). Despacho ótimo de unidades geradoras em sistemas hidrelétricos via heurística baseada em relaxação lagrangeana e programação dinâmica, Tese de doutorado, Universidade Estadual de Campinas, Campinas, Brasil.

CCEE (2007a). Regras de comercialização: Contabilização módulo 1, preço de liquidação das diferenças, Brasil.

URL: http: //www. ccee.org.br/

CCEE (2007b). Regras de comercialização: Contabilização módulo 4, energias asseguradas, Brasil.

URL: $h t t p: / / w w w . c c e e$.org.br/

Cicogna, M. A. (1999). Modelo de planejamento de operação energética de sistemas hidrotérmicos a usinas individualizadas orientado por objetos, Dissertação de mestrado, Universidade Estadual de Campinas, Campinas (SP), Brasil.

Costa, A. L. (2004). Alocação Ótima Conjunta de Energía e Reservas Operativas Baseada em Fluxo de Potência Ótimo Dinâmico, Tese de doutorado, Universidade Federal de Santa Catarina, Florianópolis, Brasil.

González, J. G., Roque, A. S., Campos, F. and Villar, J. (2007). Connecting the intraday energy and reserve markets by an optimal redispatch, IEEE transactions on power systems 22(4): 2220-2231.

Hirst, E. and Kirby, B. (1996). Electric - Power Ancillary Services, OAK Ridge National Laboratory, Energy Division, Tennessee, USA.

Jaleeli, N., VanSlyck, L. S., Ewart, D. N., Fink, L. H. and Hoffmann, A. G. (1992). Understanding automatic generation control, IEEE transactions on power systems 7(3): 1106-1122.

Kirschen, D. S. and Strbac, G. (2004). Fundamentals of Power System Economics, ISBN: 13:978-0-470-84572-1 (H/B), John Wiley \& Sons, England.

ONS (2003a). Arranjos comerciais para os serviços ancilares providos pelos agentes de geração. submódulo 14.2, Brasil.

URL: http://www. ons.org.br/

ONS (2003b). Contrato de prestação de serviços ancilares padrão. geradoras e pies, Brasil.

URL: http://www.ons.org.br/download/ contratos \_ancilares/geradoras I_pie. $p d f$

ONS (2008a). Elaboração da programação diária da operação eletroenergética. submódulo 8.1, Brasil.

URL: http: / /www.ons.org.br/

ONS (2008b). Norma de operação - controle da geração em operação normal. submódulo 10.6, Brasil.

URL: http: //www.ons.org.br/

Rebours, Y. G., Kirschen, D. S., Trotignon, M. and Rossignol, S. (2007). A survey of frecuency and voltage control ancillary services - part i: Technical features, IEEE Trans. on Power Systems 22(1): 350-357.

Rebours, Y. G., Kirschen, D. S., Trotignon, M. and Rossignol, S. (2007a). A survey of frecuency and voltage control ancillary services - part ii: Economic features, IEEE Trans. on Power Systems 22(1): 358-366.

Rebours, Y., Kirschen, D. and Trotignon, M. (2007b). Fundamental design issues in markets for ancillary services, The Electricity Journal 20(6): 26-34.

URL: http://www.sciencedirect.com/

Ribeiro, P. M. (2005). Remuneração dos Serviços Ancilares de Suporte de Potência Reativa e Reserva de Potência Quando Providos por Geradores, Dissertação de mestrado, Pontíficia Universidade Católica de Rio de Janeiro, Rio de Janeiro.

Rudnick, H., Barroso, L. A. and Bezerra, B. (2008). A delicate balance in south america, IEEE power \& energy magazine 6(4): 22-35.

Schier, J. (2007). Análise Comparativa de Metodologias de Alocação de Serviços Ancilares de Reserva em Mercados de Energia Elétrica, Dissertação de mestrado, Universidade Federal de Santa Catarina, Florianópolis, Brasil. 
Shahidehpour, M., Yamin, H. and Li, Z. (2002). Market Operations in Electric Power Systems, ISBN 0-471-44337-9, John Wiley \& Sons, New York.

Silva, F. S. (2005). Modelo de Formação de Preços para Serviços Ancilares de Reserva, Dissertação de mestrado, Universidade de Brasília, Faculdade de tecnologia, Brasil.

Silva, I. J., Rider, M. J., Romero, R. and Murari, C. A. (2005). Transmission network expansion planning considering uncertainness in demand, Power Engineering Society General Meeting, pp. 1424-1429.

Sousa, T., Jardini, J. A., Masuda, M. and de Lima, R. A. (2004). Spinning reserve service pricing in hydroelectric power plants, IEEE/PES Transmission \& Distribution Conference \& Exposition: Latin America .

Stoft, S. (2002). Power System Economics: Designing Markets for Electricity, ISBN 0-471-15040-1, IEEE / Wiley, New York.

Swider, D. J. (2007). Efficient scoring-rule in multipart procurement auctions for power system reserve, IEEE Trans. on Power Systems 22(4): 1717-1725.

Velasco, C. J. (2004). Metodologia para o Cálculo do Valor do Serviço e da Remuneração dos Agentes Fornecedores de Reservas Operativas, Tese de doutorado, Pontíficia Universidade Católica de Rio de Janeiro, Rio de Janeiro, Brasil. 